\title{
Pionic transitions of excited charmed mesons in the covariant oscillator quark model
}

\section{Tomohito MAEDA*}

Department of Science and Manufacturing Technology, Junior College Funabashi Campus, Nihon University, Funabashi 274-8501, Japan

E-mail: maeda.tomohitodnihon-u.ac.jp

\section{Kenji YAMADA}

Department of Science and Manufacturing Technology, Junior College Funabashi Campus, Nihon University, Funabashi 274-8501, Japan

E-mail: yamada.kenjidnihon-u.ac.jp

\section{Masuho ODA}

Research Institute of Science and Technology, College of Science and Technology,

Nihon University, Tokyo 101-8308, Japan

\begin{abstract}
We study the pionic transitions of orbitally excited charmed mesons by using the covariant oscillator quark model. An important feature of this model is that hadrons are treated in a manifestly covariant way hence it could deal with the retardation effect in the transition form factor. We examined the these relativistic effects for the calculated widths in comparison with other quark models. Based on the results obtained, we discuss the possible assignments for newly observed states.
\end{abstract}

XVII International Conference on Hadron Spectroscopy and Structure

25-29 September, 2017

University of Salamanca, Salamanca, Spain

\footnotetext{
*Speaker.
} 


\section{Introduction}

Spectroscopy of charmed mesons has been made a remarkable progress with recent development of high energy collider experiments [四]. Since 2010, candidates for highly excited states have

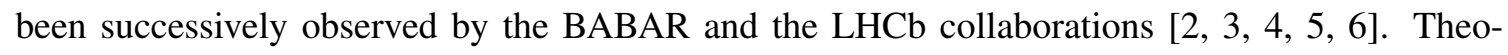
retically, as is well-known, the study of the hadronic decay is the most suitable way to probe the nature of hadrons since decay widths strongly depend on their internal structure. Along this line of

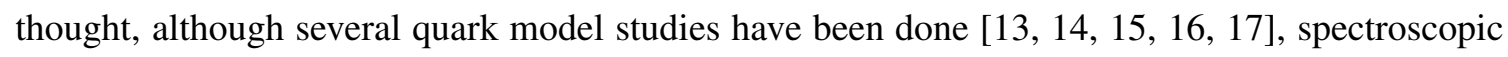
assignments for these states still remain to be completely elucidated.

In this work, we study the pionic decays of orbital excited charmed mesons by adopting the covariant oscillator quark model (COQM). An important feature of this model is that hadrons are treated in a manifestly covariant way hence it could deal with the relativistic retardation effect in the transition form factor. We examined these effects for the calculated widths in comparison with other quark models. Based on the results obtained, we discuss the possible assignments for newly observed states.

\section{Covariant Oscillator Quark Model}

The COQM [ [ $, 8,0]$ is one of the possible covariant extension of conventional non-relativistic quark model (NRQM). Its kinematical framework is known as the boosted $L S$ coupling scheme, where the internal wave functions of relevant mesons are described by a direct product of spacetime part $f\left(v^{\mu}, x^{\mu}\right)$ and spin part $W\left(v^{\mu}\right)$ and separately extended to the covariant form,

$$
\Phi(v, x)_{\alpha}^{( \pm) \beta}=f^{v_{1} v_{2} \cdots}(v, x) \otimes W_{\alpha, v_{1} v_{2} \cdots}^{\beta}(v) .
$$

The 4-dimensional simple harmonic oscillator (SHO) function which satisfies definite metric type subsidiary condition [ए]] is adopted as the space-time part, while the spin part consists of direct product of respective Dirac spinors with velocity of mesons. As a result, the squared mass spectra of mesons show a linear rising Regge trajectory, and Gaussian size parameter of the wave function is uniquely determined from the slope. The overlapping integrals of SHO functions yields the form factor $(\mathrm{FF})$,

$$
I_{\mathrm{COQM}} \sim \exp \left(-\frac{1}{4 \beta^{2}}\left(\frac{m_{Q}}{m_{q}+m_{Q}}\right)^{2}\left(q^{2}+q_{0}^{2}\right)\right) \text { at } P_{I}=0 .
$$

Here the second term in the exponent comes from relative time degree of freedom $x^{0}$, which appears as a result of covariant treatment, it could contribute to suppress the decay width, especially in the transitions with large energy transfer. In this work we treat the emitted pion as an elementary NG

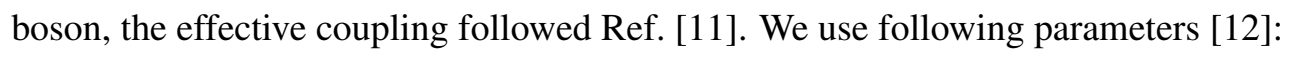

$$
\begin{aligned}
& f_{\pi}=0.130 \mathrm{GeV}, f_{K}=f_{\eta}=0.156 \mathrm{GeV}, g_{A}=0.75, \beta=0.43 \mathrm{GeV}, \\
& m_{1}=\frac{m_{\rho}}{2}=0.387 \mathrm{GeV}, m_{2}=\frac{m_{J} / \psi}{2}=1.55 \mathrm{GeV}
\end{aligned}
$$

Numerical results in comparison with experiments and other quark-models are shown in Table $\mathbb{W}$ and Table \. 
Table 1: Calculated widths for $L=0$ and $L=1$ states (in $\mathrm{MeV}$ ).

\begin{tabular}{|c|c|c|c|c|c|}
\hline State $\left({ }^{2 S+1} L_{J}\right)$ & Channel & 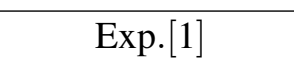 & This work & ZZ:2008[[13] & CS:2005[15] \\
\hline$D^{*}(2010)^{+}\left({ }^{3} S_{1}\right)$ & $D \pi$ & $(83.4 \pm 1.8) \cdot 10^{-3}$ & $117 \cdot 10^{-3}$ & $112 \cdot 10^{-3}$ & $52 \cdot 10^{-3}$ \\
\hline$D_{1}^{\prime}(2420)^{+}\left(j_{q}=\frac{3}{2} P_{1}\right)$ & $D^{*} \pi$ & $25 \pm 6$ & 21 & 22 & 22 \\
\hline$D_{0}^{*}(2400)^{+}\left({ }^{3} P_{0}\right)$ & $D \pi$ & $230 \pm 17$ & 264 & 248 & 283 \\
\hline$D_{1}(2430)^{0}\left(j_{q}=\frac{1}{2} P_{1}\right)$ & $D^{*} \pi$ & $384_{-110}^{+130}$ & 234 & 220 & 272 \\
\hline \multirow[t]{3}{*}{$D_{2}^{*}(2460)\left({ }^{3} P_{2}\right)$} & $D \pi$ & - & 30 & 39 & 35 \\
\hline & $D^{*} \pi$ & - & 19 & 19 & 20 \\
\hline & total & $46.7 \pm 1.2$ & 49 & 59 & 55 \\
\hline
\end{tabular}

Table 2: Calculated widths for $L=2$ states (in MeV). Here we have omitted the small contributions $(\sim 5$ $\mathrm{MeV}$ or less) from the table except for $D_{1}^{*}(2760) \rightarrow D_{1}(2430) \pi$.

\begin{tabular}{|c|c|c|c|c|c|}
\hline State $\left({ }^{2 S+1} L_{J}\right)$ & Channel & This work & Z:2010[ए4] & SCLM:2015[ㄷ] & CS:2005[ए5] \\
\hline \multirow[t]{2}{*}{$\overline{D_{1}^{*}(2760)\left({ }^{3} D_{1}\right)}$} & $D \pi$ & 89 & 156.8 & 76.13 & 73 \\
\hline & $D \eta$ & 11 & 43.2 & 9.01 & 16 \\
\hline 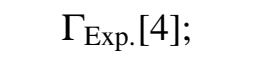 & $D_{s} K$ & 12 & 45.8 & 11.66 & 55 \\
\hline \multirow[t]{5}{*}{$177 \pm 32 \pm 20 \pm 7$} & $D^{*} \pi$ & 45 & 64.9 & 35.16 & 45 \\
\hline & $D_{s}^{*} K$ & 12 & 10.3 & 2.92 & 23 \\
\hline & $D_{1}(2430) \pi$ & 0.51 & 29.4 & 0.56 & 0.2 \\
\hline & $D_{1}{ }^{\prime}(2420) \pi$ & 201 & 187.1 & 211.72 & 189 \\
\hline & total & $\gtrsim 380$ & 553.3 & 385.06 & 523 \\
\hline$D_{3}^{*}(2760)\left({ }^{3} D_{3}\right)$ & $D \pi$ & 18.6 & 32.5 & 8.47 & 53 \\
\hline$\Gamma_{\text {Exp. }}[$ [1]]; & $D^{*} \pi$ & 16.5 & 20.6 & 7.05 & 55 \\
\hline $105 \pm 18 \pm 6 \pm 23$ & total & $\gtrsim 40$ & 67.9 & 18.07 & 277 \\
\hline
\end{tabular}

\section{Results and Discussions}

By inspecting Table 田, our model successfully reproduces experiments as well as other models concerning the $1 \mathrm{~S}$ and 1P states. Here it may be worthwhile that in Ref. [[ए3], which adopts the similar elementary-emission approach, a slightly smaller coupling constant $g_{A}=0.557$ is used. In the COQM, experiments can be reproduced with $g_{A}=0.75$, since the relativistic effect of FF suppresses the decay widths.

As may be seen in Table 凹, we may generally conclude that our calculated widths for 1D states are relatively narrow than other models, reflecting our relativistic FF. The $D_{1}^{*}(2760)$ is a plausible candidate of $1^{3} D_{1}$. Our prediction for $D_{1}^{*}(2760)$ assigned as $1^{3} D_{1}$, is much larger than the present experimental data as in other models. This indicates that the $D_{1}^{*}(2760)$ cannot be interpreted as pure $1^{3} D_{1}$ and a definite state mixing between $1 D$ - $2 S$ are required. On the other hand, the spin 3 state, $D_{3}^{*}(2760)$ is inevitably assigned to $1^{3} D_{3}$. Our predicted width for $D_{3}^{*}(2760)$ is slightly smaller than experiment. Although we do not calculate the process accompanied by $\rho / \omega$ emission, 
a considerable contribution is predicted in Ref.[ㅍ]]. Further experimental data will draw a more clear conclusion.

\section{References}

[1] C. Patrignani et al. (Particle Data Group), Chin. Phys. C, 40, 100001 (2016) and 2017 update.

[2] P. del Amo Sanchez et al. (BaBar Collaboration), Phys. Rev. D 82, 111101 (2010).

[3] R. Aaij et al. (LHCb Collaboration), JHEP 1309, 145 (2013).

[4] R. Aaij et al. (LHCb Collaboration), Phys. Rev. D91, 092002 (2015).

[5] R. Aaij et al. (LHCb Collaboration), Phys. Rev. D92, 032002 (2015).

[6] R. Aaij et al. (LHCb Collaboration), Phys. Rev. D94, 072001 (2016).

[7] S. Ishida and M. Oda, in Proceedings of Extended Objects and Bound Systems, ed. O. Hara, S. Ishida and S. Naka (World Scientific, 1992), p. 181. See also, S. Ishida and M. Oda, Prog. Theor. Phys. Suppl. No. 67 (1979), 209.

[8] S. Ishida and K. Yamada, Phys. Rev. D 35 (1987), 265.

[9] S. Ishida, K. Yamada and M. Oda, Phys. Rev. D 40, 1497 (1989).

[10] T. Takabayasi, Prog. Theor. Phys. Suppl. 67, 1 (1979).

[11] R. P. Feynman, M. Kislinger, and F. Ravndal, Phys. Rev. D 3, 2706 (1971).

[12] S. Ishida and M. Oda, Prog. Theor. Phys. 89, 1033 (1993).

[13] X.H. Zhong and Q. Zhao, Phys. Rev. D 78, 014029 (2008).

[14] X. H. Zhong, Phys. Rev. D 82, 114014 (2010).

[15] F. E. Close and E. S. Swanson, Phys. Rev. D 72, 094004 (2005).

[16] Q. T. Song, D. Y. Chen, X. Liu, and T. Matsuki, Phys. Rev. D 92, 074011 (2015).

[17] S. Godfrey and K. Moats, Phys. Rev. D 93, 034035 (2016). 